\title{
A Machine Learning Based Approach to Study Morphological Features of Bees ${ }^{+}$
}

\author{
John Yoo ${ }^{1}$, Md Zakir Hossain 1,2*(D) and Khandaker Asif Ahmed 2*(D) \\ 1 The Australian National University (ANU), ACT 2601, Australia \\ 2 The Commonwealth Scientific and Industrial Research Organisation (CSIRO), ACT 2601, Australia \\ * Correspondence: \{zakir.hossain, khandakerasif.ahmed\}@csiro.au \\ + Presented at the 1st International Electronic Conference on Entomology (IECE 2021), 1-15 July 2021; Available \\ online: https://iece.sciforum.net/.
}

Citation: Yoo, J.; Hossain, M.Z.; Ahmed, K.A. A Machine Learning Based Approach to Study Morphological Features of Bees. Proceedings 2021, 1, 0. https://doi.org/10.3390/IECE-10607

Published: 5 July 2021

Publisher's Note: MDPI stays neutral with regard to jurisdictional claims in published maps and institutional affiliations.

Copyright: (c) 2020 by the authors. Licensee MDPI, Basel, Switzerland. This article is an open access article distributed under the terms and conditions of the Creative Commons Attribution (CC BY) license (https:// creativecommons.org/licenses/by/ $4.0 /)$.

\begin{abstract}
Bees are the major pollinators of agricultural crops and due to numerous factors, the global bee population is declining drastically. Identification and extraction of numerous body features of bees can allow us to understand the population dynamics and bee-hive health of an agricultural area. Morphological key-based bee studies are well established procedures for these tasks, which are time consuming and need critical knowledge about different bees species. Recently, numerous machine learning (ML) methods have been implemented on numerous insect species, but there is a scarcity of deep learning models for morphological studies of bees. In our current study, we applied ML methods to extract variants of class activation maps that visually display distinguishing morphological features of bees. We sourced an image data set of eleven different species of Bumblebee (Bombus sp.), Honey bee (Apis sp.) and Carpenter bee (Xylocopa sp.) from iNaturalist, curated and fine-tuned against fifteen state-of-the-art image classification models. An accuracy of 93.66\% was obtained with a ResNest101e model, and including data augmentation improves the performance to the highest accuracy of $94.27 \%$. We also compared the ML extracted visual features with traditional morphological key-based features and showed existing unsupervised ML models are error prone in numerous instances due to their focus on overall features, whereas manual methods benefited by focusing only on the main discriminating body features, showing a potential scope of improvement the existing models. Overall, our model will be implicated in bee-morphology based tasks of apiculture, such as distinguishing between healthy and parasitic bees, and classification tasks of similar insect species.
\end{abstract}

Keywords: Machine learning; Bees; Morphology; Feature extraction

\section{Introduction}

Bees are the essential element of our ecosystem, contributing as primary pollinators of agricultural crops and promote the sustainable development of our ecosystem [1,2]. Approximately 25000 bee species has been reported worldwide [4]. In recent years, there is a clear declination trend in both wild and domesticated bee populations around the world, with threats including the loss of habitat, increased use of pesticides, invasive species and climate change $[15,16]$. In parallel this would result in the decline in the wild plant and crop population with the $75 \%$ of the latter mostly pollinated by bees [17]. The decline in bee populations is of concern not only to the greater ecosystem, but also in industries relying on bee products. Various industries relying on the produce from bees such as the honey and wax industries would suffer greatly. The study of bee morphology and identify them down to the species level would allow us to understand bee-hive health and population dynamics in target areas for which specific measures can be taken to maintain and promote their livelihood and impact on the greater ecosystem [3].

Traditional bee morphology studies were mainly focused on systematics of different bee species. Systematics is a prevalent task, which involves manual collection of mor- 
phometric data such as wing data and curation by a domain expert (taxonomist) [19]. Recently numerous DNA-based [20] and machine learning (ML) based [5-7] approaches had been implemented to compensate workloads in bee systematics, but there is a still lack of approach for bee morphology studies [20]. Recent advances in ML models have paved the way to accurately derive discriminating features from general images, which has potential to overcome manual curation works in morphological works. In particular, the advent of Convolutional Neural Networks (CNN) [8,9] for image analysis have produced models with identification accuracy comparable or better than humans in multiple visual recognition tasks [9-12]. CNN models achieved high accuracy $(90 \%+)$ when differentiating between closely related subordinate level categorisation using general image data [14]. DeepABIS [45] and ABIS [5] models utilised different CNNs to automatically generate features from wing images of bees to classify up to species and subspecies level, but both of the models need heavily curated wing data set. Data acquisition and feasibility for such extensive models become thus low as these curating procedures are extremely labour intensive and sometimes impossible depending on the number of bee species. Although the imaging of bees wings are few and specific, there are a number of extensive public data set of general images of bees with diverse backgrounds. A image-based ML model, capable of successfully identifying bees and their distinguishing morphological features, would allow us to measure population dynamics more quickly and conveniently, levels of decline and thus empower us with the information to generate conservation strategies.

In this paper, we explore the task of bee morphology study with various deep learning models and qualitatively analyse the effectiveness of class activation maps from the best performing model on extracting distinguishing features from our chosen eleven bee species. A successful extraction of distinguishing morphological features from the class activation maps would allow us to fine-tune ML models to achieve better results on classification tasks with the same population of bee species, on top of this, we can gather valuable data to explore what a machine learning model considers the distinguishing differences between bees in a particular subset of species.

\section{Methodology}

To briefly describe our experimental design, we collected bee images from public domain, studied the key morphological features from existing literature and compared the features with ML extracted features to determine the discrepancy between traditional and state-of-art ML methods and probable solution to enhance the model accuracy for studying bee morphology. To extract ML extracted features, we used numerous ML models to classify different bee species, identified two best performed models, enhanced their performance using data augmentation and implemented class activation map (CAM).

\subsection{Data collection and pre-processing}

The images of current study were sourced from iNaturalist.org, which contains images of different organisms, with $92.3 \%$ to $97.3 \%$ proper taxonomic annotations or classifications [37]. We focused on the major three bee genera around the world, namely - Apis, Xylocopa and Bombus. Bees of Apis and Bombus genera are known as honey bees and bumble bees, well known for producing honey and wax, whereas bees of Xylocopa genus are called carpenter bees, which do not produce honey and some are known as parasitic to wood plants. From these three genera, we choose top eleven species based on the number of available images and selected following species: A. mellifera, $X$. virginica, X. micans, $X$. sonorina, X. tabaniformis, X. violacea, B. griseocollis, B. impatiens, B. pensylvanicus, B. terrestris, and $B$. vosnesenskii. Images were downloaded in bulk using an in-house python script, and the number of images were balanced based on the lowest available images of $X$. micans. Finally the data set contains a total of 24,695 images of bees, with 2,245 images of each species. For pre-processing, we resized the images to $224 \times 224$ pixels and normalised to fit into pre-trained ImageNet models. From this processed dataset, $80 \%$ and $20 \%$ was used for training and testing purpose. 


\subsection{Traditional Key Features}

We collected traditional key morphological features of the 3 genera obtained from dichotomous keys and related genera studies [34-36]. In particular, we focused mainly on distinguishing visual features (e.g. body coloration, body shape, size, wing features), which can be captured in visual images, and models can use them as classifiers for classification.

Table 1: Major morphological features of three bee genera [34-36].

\begin{tabular}{|c|c|c|c|}
\hline $\begin{array}{l}\text { Genus/ } \\
\text { Features }\end{array}$ & Apis & Bombus & Xylocopa \\
\hline Body size & 7 to $19 \mathrm{~mm}$ & 10 to $40 \mathrm{~mm}$ & 13 to $30 \mathrm{~mm}$ \\
\hline Body shape & Elongated & Broad and stout & Elongated \\
\hline Body hair & Moderately hairy & $\begin{array}{l}\text { Densely long-haired } \\
\text { especially towards } \\
\text { the apex }\end{array}$ & $\begin{array}{l}\text { Moderately hairy } \\
\text { with black hairs }\end{array}$ \\
\hline Body bands & $\begin{array}{l}\text { Several abdominal } \\
\text { grey bands }\end{array}$ & Fewer to none bands & Usually no bands \\
\hline Body coloration & Yellow to grey & $\begin{array}{l}\text { Black to yellow, with } \\
\text { black furr }\end{array}$ & Black to yellow \\
\hline Wing coloration & Clear & $\begin{array}{l}\text { Clear to smoky } \\
\text { brownish }\end{array}$ & $\begin{array}{l}\text { Strongly purplish- } \\
\text { iridescent }\end{array}$ \\
\hline Eye & Hairy eyes & Bare eyes & Hairy eyes \\
\hline Leg features & $\begin{array}{l}\text { Hind tibilia spurs ab- } \\
\text { sent }\end{array}$ & $\begin{array}{l}\text { Hind tibilia spurs } \\
\text { present }\end{array}$ & $\begin{array}{l}\text { Hind tibilia spurs } \\
\text { present, hairy hind } \\
\text { leg }\end{array}$ \\
\hline Hind wing & $\begin{array}{l}\text { Jugal lobe absent, } \\
\text { strong venation }\end{array}$ & Jugal lobe absent & $\begin{array}{l}\text { Jugal lobe present, } \\
\text { strongly papillated }\end{array}$ \\
\hline $\begin{array}{l}\text { Wing marginal } \\
\text { cells }\end{array}$ & Long and narrow & Shorter and broader & $\begin{array}{l}\text { Very long with } \\
\text { rounded apex }\end{array}$ \\
\hline Wing stigma & $\begin{array}{l}\text { Small and slender, } \\
\text { shorter than pres- } \\
\text { tigma }\end{array}$ & $\begin{array}{l}\text { Wider than pres- } \\
\text { tigma }\end{array}$ & $\begin{array}{l}\text { No stigma with very } \\
\text { long prestigma. }\end{array}$ \\
\hline
\end{tabular}

\subsection{Models for classification and feature extraction}

We implemented eleven state-of-the-art deep learning models, which were pretrained with ImageNet for fine-grained image classification tasks, namely EfficientNet[21], ResNest101e[27], CSPDarkNet[28], TresNet[22], RexNet[23], CSPResNet[29], NoisyStudent[26], Vision Transformer[24], LCA-CNN[42], SpinalNet[32], and RegNet[31].

These models were executed using either a modified version of Wightman's pytorchimage-models [25] or a direct code implementation. Further, all layers in the models were fine-tuned for our bee dataset. The deep networks were trained using a mini-batch stochastic gradient descent optimiser with a batch size of 32. Learning rate, momentum, and weight decay were kept at $0.01,0.9$, and 0.0001 respectively. We also employed a dropout[43] value of 0.2 to prevent overfitting. Each model was executed for a total of 100 epochs with a NVIDIA 2070 RTX GPU with 8gb onboard memory. Further, for data augmentation, we applied RandAugment [33] with a 50\% chance and a transformation magnitude of 9 to the best two performing models onto our dataset. We utilised the gradients within the best performing model to obtain a Gradient-weighted Class Activation Mapping (Grad-CAM [44]) which visually indicates the discriminative region used by the model to classify bee species. We used a modified Grad-CAM++[38] which outperformed than the original Grad-CAM on providing visual explanations for deep learning models. 


\section{Results}

Our first step was to determine and train a deep learning model on our bee data set in order to obtain a machine-based representation of each class. We experimented on eleven different state-of-the-art deep learning models for image classification in order to determine the best architecture for our bee task. Major distinguishing features of three bee genera has been shown in Table 1.Further, figure 1 illustrates the classification accuracies obtained based on different CNN architecture families. Models of EfficientNet, ResNet and other families got accuracies of $92.13 \pm 0.75 \%, 91.57 \pm 1.13 \%, 89.21 \pm 1.22 \%$ respectively.
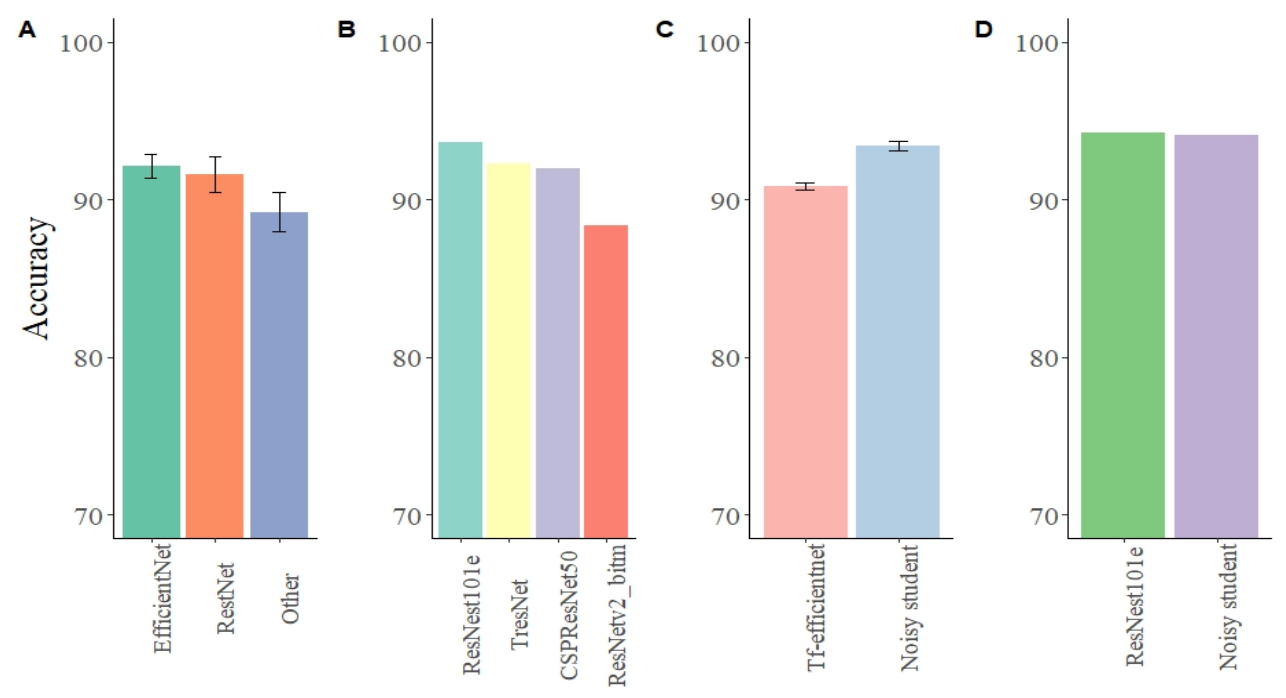

Figure 1. Classification accuracies across (A) different model families, (B) different ResNest models, (C) different efficientNet models and (D) best two models with data augmentation

From the ResNest family, ResNet101e, Tresnet, CSPResNet50 and ResNetv2 got accuracies of $93.66 \%, 92.25 \%, 92 \%, 88.38 \%$ respectively. Models of EfficientNet families outperformed all of the models. Tfefficient got accuracy of $90.86 \%$, whereas with the inclusion of noisy student, it improved to $93.41 \%$. Overall, among the eleven models, ResNest and TFefficient Net with noisy student best performed on highest accuracy for bee classification. We used state-of-art data augmentation methods on these top two models to boost their classification performance. We have found that the performance of both models improved as a result of RandAugment, where ResNest101e and TFefficient Net with noisy student models resulted in the accuracy of $94.27 \%$ and $94.07 \%$ respectively. For CAM, we used the best performed ResNest101e model to retrieve features from bee dataset with highest confidence (Figure 2). A qualitative analysis of the images compared to the traditional morphological features of their genera showed us - For Apis and Bombus, the ResNest101e model mainly focused on the abdomen, lower parts of the head and also able to capture both the body colouration and strips. on the other hand, for Xylocopa, the model focused more on the wings and head portions of the bee with CAMs often following the shape of the wings.

\section{Discussion}

In our research we have aimed to produce a modified model capable of producing visual maps displaying distinguishing features between bees spanning three genera and eleven species. Using a modified ResNest model and data augmentation, we got $94.27 \%$ classification accuracy. Further, Grad-CAM++ implementation on our model resulted in CAMs of the best samples images indicative of their respective class. With a qualitative analysis and comparison of the CAMs against traditional key morphological features for each genus we find that the CAMs produced are insufficient in fine-tuning and discovering key features between each class. 


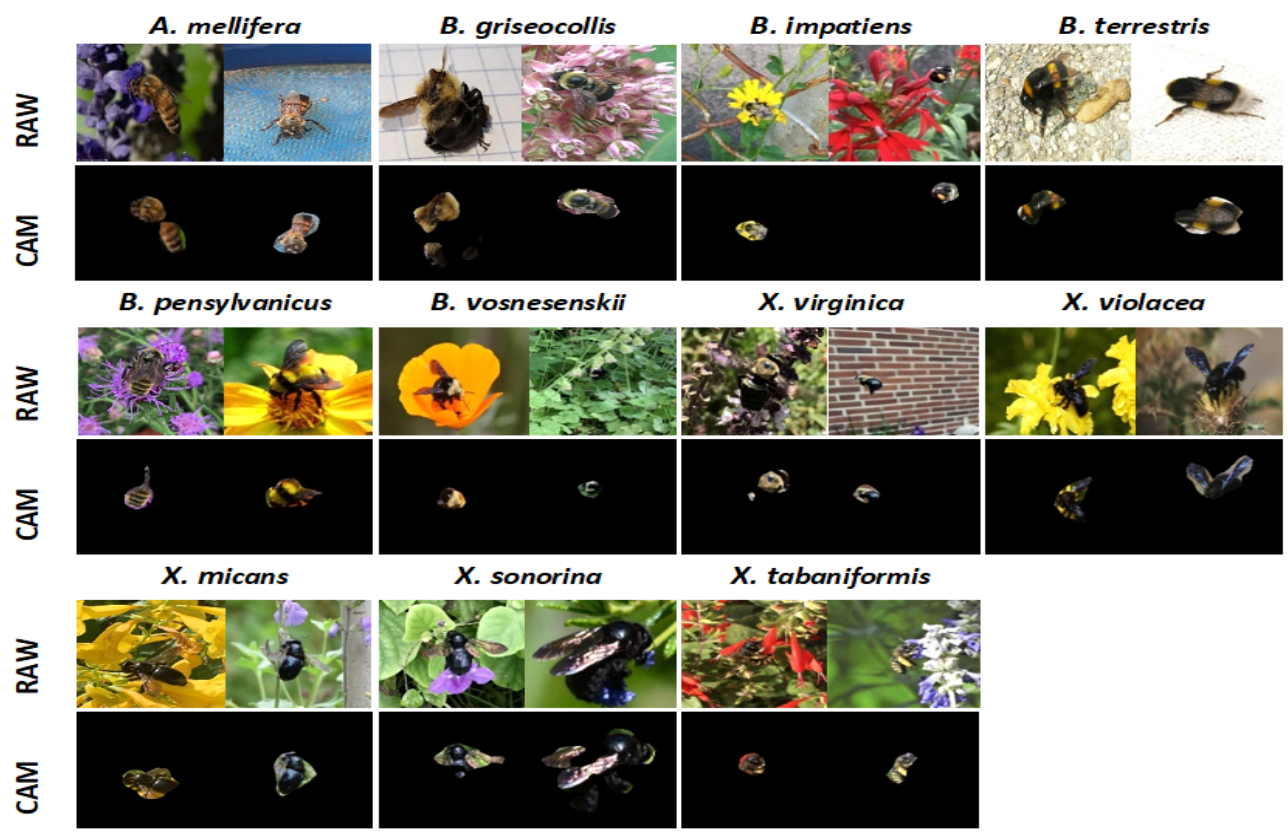

Figure 2. Raw and CAM images of different bees species resulted from our model

ResNest is able to outperform EfficientNet based models on various object detection and segmentation transfer learning tasks as well as image classification on ImageNet [39]. Our modified ResNest model well-suited for fine grained classification task of bee species, compared to other state of the art models as it achieves the highest classification accuracy of $94.27 \%$. In our experiments we also explore the effects of model scaling with regards to classification performance improvement for our task. On the topic of ResNest, despite the lower amount of pre-trained data the split attention blocks potentially work well to improve learned feature representations for our task, with the scaled depth helping to learn complex features of the bees such as wings. An analysis of the models leads us to the following conjectures regarding their high performance against the bees database. First we can compare the usage of pre-trained data in each model, both NoisyStudent and TensorFlow EfficientNet use JFT-300M and ImageNet as opposed to a majority of the other tested models including ResNest. Naturally this increases the performance of the models. Comparing the performances of the less scaled models (e.g. tf-efficientnet-b0 and ResNest50d), we have found that, scaling up the image resolution, depth and width provide accuracy boosts. Scaling of all three dimensions provides larger improvements to the accuracy as opposed to the singular depth adjustment in ResNest. Despite this, we can see that the modular split attention blocks in ResNest are much more effective for a fine-grained classification task. With regards to NoisyStudent we can see from the improvement in accuracy compared to the base tf-EfficientNet-b3 model that potentially the forceful generalisation of the student teacher model helped with the small feature representations in the bee database.

Previous works on classifying bees such as DeepABIS [45] and ABIS [5] have focused on images of bees in controlled environments and mainly on their wing data. The focus on wing data aligns with our findings of traditionally recognised distinguishing features of bees in Table 1, which, along with the controlled lab imaging environments explains their relatively high classification performance. As it is often difficult to inspect fine features of bee wings in our general image data set, there has been a larger focus of our model to distinguish based on other largely visible body features and this can be identified in the CAM images. Thenmozhi et al. [40] uses a ResNet101 model on a data set of 40 insect species and achieves an accuracy of $93.99 \%$. With a larger data set and scope of insect species we can attribute the success of the model cropped and zoomed in images of the insects in the data set as opposed to the general images in our study. Nguyen et al. [41] 
achieves its highest accuracy of $95.52 \%$ with an EfficientNetb3 model on a data set of 4,449 images of 5 different insect families (Ladybird, Mosquito, Grasshopper, Butterfly, Dragonfly). The large number of differences between these insects is a large factor in the success of their model, in comparison, with our data set of eleven different bee species with complex backgrounds, we still achieve similar accuracies with an EfficientNetb3 model $(93.136 \%)$. From our analysis of traditional morphological features in Table 1, we have found wing features are the most discriminatory, however, as the images in our data set do not have normalised imaging conditions and are often of low quality, our trained models were focused mainly on larger features as can be seen in the CAM images.

Comparatively, our study explores the distinguishing visual features that can be extracted from the models, in particular we analysed the Grad-CAMs based on our best performing model ResNest101e. The CAMs, resulted from our study, are not visually specific enough to conduce fine-grained features as distinguishing features of a bee genus. However, while this is the case, our experiments show that more general areas of a bees body can be successfully distinguished between classes despite the vastly imbalanced nature of user-images. For classes between the Apis and Xylocopa genus this can be more than enough to distinguish two samples, however, for bees with overlapping features such as species within the Xylocopa and Bombus genus, the visual features extracted by the CAM may prove to be insufficient. The inefficiency of the interpretability of the CAMs for our task can be identified by the lack of feature knowledge by the deep learning models. While to us humans morphological features of bees can be identified visually from images, it is difficult for a model to learn and categorise these features from a single class labelled image.

\section{Conclusion}

In our study, we explored the morphology of bee species and proposed the usage of Class Activation Maps to obtain visual indicators of distinguishing features. We constructed a data set of 24,695 bee images spanning eleven species and three bee genera, used different state-of-the-art deep learning model architectures and obtained the highest accuracy of $94.27 \%$ with a ResNest101e model following data augmentation. From an analysis of samples with the highest model confidence we conduce that CAMs are sufficient to highlight general areas of bees that are enough to distinguish species such as in the Apis and Xylocopa genera, however, insufficient for those with many overlapping features. A key limitation in our approach is the lack of defined feature annotations for each input image which stifles the deep learning models ability to learn key features. A lack of computational processing power also meant that we were unable to explore further on a larger set of classes. Future work could be conducted on a smaller set of annotated bee images outlining morphological features of each bee in order to obtain more robust classification and visual results. Additional modified CAM approaches could also be considered such as applying a majority membership alongside annotated feature areas of interest.

\section{References}

1. Calderone NW (2012) Insect Pollinated Crops, Insect Pollinators and US Agriculture: Trend Analysis of Aggregate Data for the Period 1992-2009. PLoS ONE 7(5): e37235. https://doi.org/10.1371/journal.pone.0037235

2. Matias, D. M., Leventon, J., Rau, A. L., Borgemeister, C., and von Wehrden, H. (2017). A review of ecosystem service benefits from wild bees across social contexts. Ambio, 46(4), 456-467. https://doi.org/10.1007/s13280-016-0844-z

3. Brown, M.J.F., Paxton, R.J. The conservation of bees: a global perspective. Apidologie 40, 410-416 (2009). https:// doi.org/10.1051/apido/2009019

4. Potts SG, Biesmeijer JC, Kremen C, Neumann P, Schweiger O, Kunin WE. Global pollinator declines: Trends, impacts and drivers. Trends in Ecology \& Evolution. (2010);25:345-353. doi: 10.1016/j.tree.2010.01.007

5. Arbuckle, T., Schröder, S., Steinhage, V., and Wittmann, D. (2001, October). Biodiversity informatics in action: identification and monitoring of bee species using ABIS. In Proc. 15th Int. Symp. Informatics for Environmental Protection (Vol. 1, pp. 425-430). ETH Zurich. 
6. De Moor, B., De Gersem, P., De Schutter, B., \& Favoreel, W. (1997). DAISY: A database for identification of systems. JOURNAL A, 38, 4-5.

7. Úna Fitzpatrick, Tomás E. Murray, Robert J. Paxton, John Breen, Don Cotton, Veronica Santorum, Mark J.F. Brown, Rarity and decline in bumblebees - A test of causes and correlates in the Irish fauna, Biological Conservation, Volume 136, Issue 2, 2007, Pages 185-194, ISSN 0006-3207, https://doi.org/10.1016/j.biocon.2006.11.012.

8. A. Krizhevsky, I. Sutskever, and G. Hinton. Imagenet classification with deep convolutional neural networks. In NIPS, 2012.

9. D. Ciresan, U. Meier, and J. Schmidhuber. Multi-column deep neural networks for image classification. In CVPR, 2012.

10. Y. Taigman, M. Yang, M. Ranzato, and L. Wolf. Deepface: Closing the gap to human-level performance in face verification. In CVPR, 2014.

11. Y. Sun, Y. Chen, X. Wang, and X. Tang. Deep learning face representation by joint identificationverification. In NIPS, 2014.

12. L. Wan, M. Zeiler, S. Zhang, Y. L. Cun, and R. Fergus. Regularization of neural networks using dropconnect. In ICML, pages 1058-1066, 2013.

13. Barré, P., Stöver, B. C., Müller, K. F., and Steinhage, V. (2017). LeafNet: A computer vision system for automatic plant species identification. Ecological Informatics, 40, 50-56.

14. Zhongqi Lin, Jingdun Jia, Wanlin Gao, Feng Huang, Fine-grained visual categorization of butterfly specimens at sub-species level via a convolutional neural network with skip-connections, Neurocomputing, Volume 384, 2020, Pages 295-313, ISSN 0925-2312, https:// doi.org/10.1016/j.neucom.2019.11.033.

15. Kremen C, Williams NM, Aizen MA, Gemmill-Herren B, LeBuhn G, Minckley R, Packer L, Potts SG, et al. Pollination and other ecosystem services produced by mobile organisms: A conceptual framework for the effects of land-use change. Ecology Letters. 2007;10:299-314. doi: 10.1111/j.1461-0248.2007.01018.x.

16. Chagnon, M., Kreutzweiser, D., Mitchell, E.A. et al. Risks of large-scale use of systemic insecticides to ecosystem functioning and services. Environ Sci Pollut Res 22, 119-134 (2015). https://doi.org/10.1007/s11356-014-3277-x

17. Pettis, J.S., Delaplane, K.S. Coordinated responses to honey bee decline in the USA. Apidologie 41, 256-263 (2010). https:/ / doi.org/10.1051/apido/2010013

18. Klein Alexandra-Maria, Vaissière Bernard E, Cane James H, Steffan-Dewenter Ingolf, Cunningham Saul A, Kremen Claire and Tscharntke Teja 2007Importance of pollinators in changing landscapes for world cropsProc. R. Soc. B.274303-313 http://doi.org/10.1098/rspb.2006.3721

19. Schroder, S., Wittmann, D., Drescher, W., Roth, V., Steinhage, V., \& Cremers, A. B. (2002). The new key to bees: automated identification by image analysis of wings. Pollinating bees-the Conservation Link Between Agriculture and Nature, Ministry of Environment, Brasilia, 209-218.

20. Jason Gibbs. DNA barcoding a nightmare taxon: assessing barcode index numbers and barcode gaps for sweat bees. Genome. (2017) 61(1): 21-31. https:/ / doi.org/10.1139/gen-2017-0096

21. Tan, M., \& Le, Q. (2019, May). Efficientnet: Rethinking model scaling for convolutional neural networks. In International Conference on Machine Learning (pp. 6105-6114). PMLR.

22. Ridnik, T., Lawen, H., Noy, A., Ben Baruch, E., Sharir, G., \& Friedman, I. (2021). Tresnet: High performance gpu-dedicated architecture. In Proceedings of the IEEE/CVF Winter Conference on Applications of Computer Vision (pp. 1400-1409).

23. Han, D., Yun, S., Heo, B., \& Yoo, Y. (2020). Rexnet: Diminishing representational bottleneck on convolutional neural network. arXiv preprint arXiv:2007.00992.

24. Dosovitskiy, A., Beyer, L., Kolesnikov, A., Weissenborn, D., Zhai, X., Unterthiner, T., ... \& Houlsby, N. (2020). An image is worth 16x16 words: Transformers for image recognition at scale. arXiv preprint arXiv:2010.11929.

25. PyTorch Image Models, Ross Wightman, 2019, GitHub, GitHub Repository, https://github. com/rwightman/pytorch-image-models

26. Xie, Q., Luong, M. T., Hovy, E., \& Le, Q. V. (2020). Self-training with noisy student improves imagenet classification. In Proceedings of the IEEE/CVF Conference on Computer Vision and Pattern Recognition (pp. 10687-10698).

27. Zhang, H., Wu, C., Zhang, Z., Zhu, Y., Zhang, Z., Lin, H., ... \& Smola, A. (2020). Resnest: Split-attention networks. arXiv preprint arXiv:2004.08955.

28. Bochkovskiy, A., Wang, C. Y., \& Liao, H. Y. M. (2020). Yolov4: Optimal speed and accuracy of object detection. arXiv preprint arXiv:2004.10934. 
29. Wang, C. Y., Liao, H. Y. M., Wu, Y. H., Chen, P. Y., Hsieh, J. W., \& Yeh, I. H. (2020). CSPNet: A new backbone that can enhance learning capability of CNN. In Proceedings of the IEEE/CVF conference on computer vision and pattern recognition workshops (pp. 390-391).

30. Kolesnikov, A., Beyer, L., Zhai, X., Puigcerver, J., Yung, J., Gelly, S., \& Houlsby, N. (2019). Big transfer (bit): General visual representation learning. arXiv preprint arXiv:1912.11370, 6(2), 8.

31. Radosavovic, I., Kosaraju, R. P., Girshick, R., He, K., \& Dollár, P. (2020). Designing network design spaces. In Proceedings of the IEEE/CVF Conference on Computer Vision and Pattern Recognition (pp. 10428-10436).

32. Kabir, H. M., Abdar, M., Jalali, S. M. J., Khosravi, A., Atiya, A. F., Nahavandi, S., \& Srinivasan, D. (2020). Spinalnet: Deep neural network with gradual input. arXiv preprint arXiv:2007.03347.

33. Cubuk, E. D., Zoph, B., Shlens, J., \& Le, Q. V. (2020). Randaugment: Practical automated data augmentation with a reduced search space. In Proceedings of the IEEE/CVF Conference on Computer Vision and Pattern Recognition Workshops (pp. 702-703).

34. Michener, C. D., McGinley, R. J., \& Danforth, B. N. (1994). The bee genera of North and Central America (Hymenoptera: Apoidea). Smithsonian Institution Press.

35. Karunaratne, W. A. I. P., \& Edirisinghe, J. P. (2008). Keys for the identification of common bees of Sri Lanka. Journal of the National Science Foundation of Sri Lanka, 36(1), 69-89.

36. Michener, C. D. (2000). The bees of the world (Vol. 1). JHU press.

37. Shem Unger, Mark Rollins, Allison Tietz \& Hailey Dumais (2020) iNaturalist as an engaging tool for identifying organisms in outdoor activities, Journal of Biological Education, DOI: 10.1080/00219266.2020.1739114

38. Chattopadhay, A., Sarkar, A., Howlader, P., \& Balasubramanian, V. N. (2018, March). Gradcam++: Generalized gradient-based visual explanations for deep convolutional networks. In 2018 IEEE Winter Conference on Applications of Computer Vision (WACV) (pp. 839-847). IEEE.

39. Deng, J., Dong, W., Socher, R., Li, L. J., Li, K., \& Fei-Fei, L. (2009, June). Imagenet: A largescale hierarchical image database. In 2009 IEEE conference on computer vision and pattern recognition (pp. 248-255). Ieee.

40. Thenmozhi, K., \& Reddy, U. S. (2019). Crop pest classification based on deep convolutional neural network and transfer learning. Computers and Electronics in Agriculture, 164, 104906.

41. Nguyen, T. T., Vien, Q. T., \& Sellahewa, H. (2021). An efficient pest classification in smart agriculture using transfer learning. EAI Endorsed Transactions on Industrial Networks and Intelligent Systems, 8(26), e1.

42. Youxiang Zhu, Ruochen Li, Yin Yang, Ning Ye, Learning Cascade Attention for fine-grained image classification, Neural Networks, Volume 122, 2020, Pages 174-182, ISSN 0893-6080, https://doi.org/10.1016/j.neunet.2019.10.009.

43. Srivastava, N., Hinton, G., Krizhevsky, A., Sutskever, I., \& Salakhutdinov, R. (2014). Dropout: a simple way to prevent neural networks from overfitting. The journal of machine learning research, 15(1), 1929-1958.

44. Selvaraju, R. R., Cogswell, M., Das, A., Vedantam, R., Parikh, D., \& Batra, D. (2017). Grad-cam: Visual explanations from deep networks via gradient-based localization. In Proceedings of the IEEE international conference on computer vision (pp. 618-626).

45. Buschbacher, K., Ahrens, D., Espeland, M., \& Steinhage, V. (2020). Image-based species identification of wild bees using convolutional neural networks. Ecological Informatics, 55, 101017. 